INTERVIEW

For reprint orders, please contact: reprints@futuremedicine.com

\section{Four steps to eliminate or reduce pain in children caused by needles (part 1)}

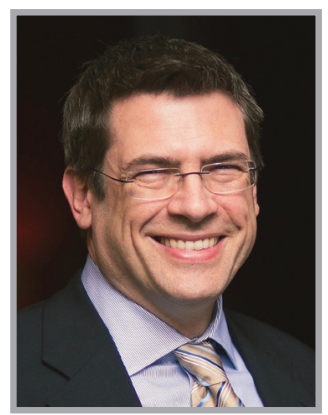

Dr Stefan Friedrichsdorf* speaks to Jade Parker, Commissioning Editor: Stefan J Friedrichsdorf, MD, is medical director of the Department of Pain Medicine, Palliative Care and Integrative Medicine at Children's Hospitals and Clinics of Minnesota, Minneapolis/St Paul, MN, USA, home to one of the largest and most comprehensive programs of its kind in the country. The interdisciplinary pain team is devoted to prevent and treat acute, procedural, neuropathic, psycho-social-spiritual, visceral, and chronic/complex pain for all inpatients and outpatients in close collaboration with all pediatric subspecialties at Children's Minnesota. The palliative care team also provides holistic care for pediatric patients with life-threatening diseases and adds an extra layer of support to the care of children with serious illness and their families. Integrative medicine provides and teaches integrative ('non-pharmacological') therapies, such as massage, acupuncture/acupressure, biofeedback, aromatherapy and self-hypnosis, to provide care that promotes optimal health and supports the highest level of functioning in all individual children's activities. Children's Minnesota became the first children's hospital to system-wide implement a "Children's Comfort Promise: We promise to do everything to prevent and treat pain," resulting in decrease or elimination of needle pain caused by vaccinations, blood draws, intravenous access, and injections in more than 200,000 children annually.

First draft submitted: 11 November 2016; Accepted for publication: 17 November 2016; Published online: 30 November 2016

Q Children's Minnesota's Department of Pain Medicine, Palliative Care \& Integrative Medicine is one of the largest programs of its kind the USA \& won the American Pain Society Clinical Centers of Excellence Award in 2013. It recently became the first children's hospital, which after internal roll-out at four pilot organizations, received the prestigious ChildKind certification. ChildKind is a global initiative aimed at reducing pain \& unnecessary suffering in children by offering a special designation to those facilities that have demonstrated an institutional commitment to pain relief \& by providing the technical support to achieve that goal. In your opinion what is it about the center that makes it stand out so distinctly?

According to the 2010 Declaration from Montréal, access to pain management is a fundamental human right and it represents a human right violation not to treat pain [1]. We have learned in our field and here at Children's Minnesota that providing excellent

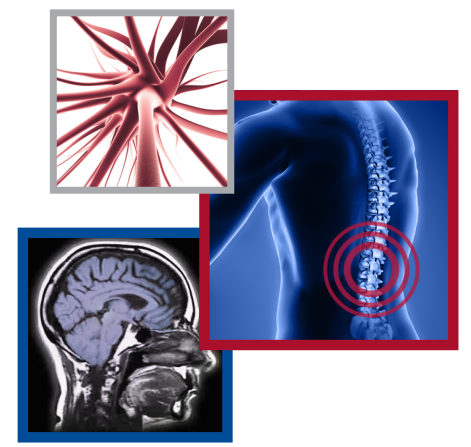

\section{KEYWORDS}

- analgesia $\bullet$ integrative strategies

- pediatric pain management

*Department of Pain Medicine, Palliative Care \& Integrative Medicine, Children's Hospitals and Clinics of Minnesota, Minneapolis, MN, USA

and

Department of Pediatrics, University of Minnesota, Minneapolis, MN, USA

stefan.friedrichsdorf@childrensmn.org

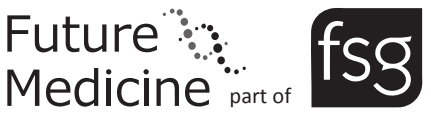


pain prevention and treatment in babies, toddlers, school kids and teenagers is so much more than just prescribing medication. Thanks to our organization's leadership and interdisciplinary pediatric care team members, we developed a very unique program at our institution. Now, we are able to utilize multimodal analgesia that is, many different modalities concurrently, to achieve optimal pain control with the least amount of side effects in children.

At Children's Minnesota, we are using advanced pharmacology, aiming to use the right amount of the right medication via the best route of administration. This may mean using simple analgesia and/or adjuvants and/or opioids, which not infrequently means rotating from one opioid to another. We have also found that advanced pain control commonly includes the integration of rehabilitation, physical therapy and exercise, occupational therapy, child life, psychology, including cognitive behavioral therapy and, most importantly, active integrative 'non-pharmacological' modalities. We are proud to have one of the largest integrative medicine programs in the USA and as such we teach children and their caregivers both inpatient and in our clinic many modalities including deep breathing, progressive muscle relaxation, self-hypnosis and biofeedback. In addition, we offer aromatherapy, massage, acupuncture and acupressure [2]. All in all, this whole package has shown that we can provide much better pain control, and when we started regular audits, we were able to demonstrate how we successfully implemented a systemwide change toward better analgesia and higher patient satisfaction.

In 2013, when we received the Center of Excellence Award from the American Pain Society, I believe the panel recognized that, on the one hand, we are a rather active pain service, which takes care of not only children with acute pain but also with procedural, chronic, psychosocial-spiritual and neuropathic pain. As part of the above-mentioned multimodal analgesia, we are also utilizing pharmacological modalities and anesthetic interventions including neuroaxial anesthesia such as epidural or paravertebral analgesia and nerve blocks, as well as using an interdisciplinary pain clinic model.

Additionally, our advocacy and training was recognized. By running the international pediatric pain master class [3], the largest pediatric pain course of its kind annually in Minneapolis and after having trained more than 500 physicians and nurse practitioners from more than 30 countries, we have shown that we are not only interested in providing excellent pain care here in our area but that we are also very interested in teaching and providing this knowledge nationally and internationally. In addition, we have trained more the 450 professional 'Trainers' through the comprehensive Education in Palliative and Endof-Life Care (EPEC) - Pediatrics curriculum, made possible by a US $\$ 1.6$ million grant by the NIH/National Cancer Institute [4].

Children's Minnesota recently received the prestigious ChildKind designation [5] certifying that we were able to demonstrate an institutional commitment to pain relief and provide the technical support to achieve that goal. Our pain service is fully integrated within the hospital, and all employees including nurses, social workers, clinicians, physicians, physiotherapists, among others, have access to modalities to provide best pain prevention and treatment. Importantly, our hospital leadership has made this a priority in patient care and made it part of the institution's strategic goals.

Q Children's Minnesota, as one of the largest US children's hospitals, is the first hospital worldwide, which implemented a 'Comfort Promise: We do Everything Possible to Prevent \& Treat Pain'; what are your four 'non-negotiables', which are now offered system wide for all children to prevent or reduce needle pain caused by vaccinations, injections, intravenous access or blood draws? To the best of our knowledge, we became the first children's hospital in the world to implement the Comfort Promise: "We will do everything possible to prevent and treat pain" [6]. The Comfort Promise [7] is applicable system-wide for all pediatric inpatients, as well as in the emergency rooms, outpatient surgical centers, outpatient laboratories and at all of our 29 primary and ambulatory clinics, aiming to eliminate or reduce all needle pain.

We are using 'lean methodology' for quality improvement in our institution. Lean improvement systems are focused on removing waste from work processes, and pain was defined as 'waste'. When a quality improvement process spans multiple areas of authority, such as units and departments, and requires extensive coordination, it is referred to as a 'Value Stream'. Therefore, the first step was to ask the children we were treating "What do we have to 
do better?" and of course, if you ask children what are you most afraid of when coming to see a doctor, whether this is in a clinic or emergency room or children's hospital, hands down the answer is "I am afraid of needle pokes or pricks." The children in our hospital told us in a system-wide survey that the least well-controlled and most distressing pain was caused by needles [8]. We then found strong evidence to offer four modalities [9-13] concurrently to eliminate or reduce needle pain, modalities we now call 'the four non-negotiables'. Using this evidence, we became the first hospital which system wide not only offers those four modalities to every child for elective needle procedures, including for vaccinations, blood draws, intravenous cannulation and injections without exception, but also audits this regularly [6].

The first non-negotiable is to 'numb the skin' using topical anesthesia. The reason we choose lidocaine $4 \%$ cream is that it is available over the counter in the USA without a prescription and it works within $30 \mathrm{~min}$ as opposed to other creams such as EMLA cream, which takes around $60 \mathrm{~min}$. Even if the needle stick infiltrates the tissue deeper than the depth of the numbed area, such as in a vaccination, there is clear evidence that numbing the skin already significantly reduces the pain and cannot be omitted.

We offer to numb the skin of any child we are doing injections for. In our critical care areas, when we may not have the time to wait $30 \mathrm{~min}$, we then use a carbon dioxide-powered, needleless lidocaine injector, similar to a 'hypospray' in Star Trek, which works within $10 \mathrm{~s}$.

Second, we use a few drops of $24 \%$ sucrose, which is sugar water, or we allow breastfeeding while we are undertaking the vaccinations or injections for infants of $0-12$ months of age. Excellent evidence has shown that this significantly reduces pain in children for needle procedures.

Third, it is important to not hold children down for needle procedures and vaccinations. If you ask children would they rather be held down or would they like to sit on their mother's lap, not surprisingly not a single child wants to be held down. Research has shown that the use of restraint is never supportive. Restraining children for procedures makes them feel ashamed, humiliated, powerless, and children report they feel having lost the right to control their own body [14]. In our institution, a posted letter signed by our chief executive officer, chief medical officer, chief nursing officer and chief operating officer states that we are offering the 'four non-negotiables' to all children, all of the time. This letter included the sentence "We at Children's Minnesota will not physically hold children down for routine needle procedures," indicating tremendous hospital leadership support for the implementation of the Comfort Promise. We are the first hospital in the USA that has not only stated that we are not going to hold down children anymore for elective needle procedures, which affects more than 200,000 children annually in our institution alone, but also undertakes regular audits. Rare exceptions would include injections required to save a child's life in an emergency. For example, if a child is presenting in the emergency room with a severe meningococal infection and requires intravenous antibiotics in minutes, then there may not be time to implement all aspects of the Comfort Promise. Of course, in that case we do everything we can to save the life of the child. But $99 \%$ of the needle pokes we are performing in our hospital are elective procedures and we have time to implement a protocol. For infants younger than 6 months, who are too young to sit up, we swaddle them in a blanket or use facilitated tucking or parental skin-to-skin contact. Whereas, kids who are older than 6 months, depending on their age, usually want to sit either on their parents lap, next to them, or alone by themselves.

Fourth, we always use age-appropriate use distraction. For an infant we may provide sounds, for a small toddler we have electrical toys which spin or make sounds. For slightly older kids we may use bubbles, pin wheels, 'I spy' pictures, and many older kids bring smartphones to provide their own form of distraction [15].

\section{Q What are your results?}

Not surprisingly, the 'Comfort Promise' represented a significant culture change in our institution. No hospital has undertaken this before on such a large level for all children, all of the time, including vaccinations, with regular audits. Our outcomes have been overwhelmingly positive; our patients' and their parents' satisfaction has significantly improved and our waiting times for blood draws have actually decreased. So carrying out all these modalities means that children are usually not crying anymore in the parking lot, in the waiting room and while the blood draw is being carried out. For our institution, and the more than 200,000 children annually who 
used to experience needle pain in the past, this is a game changer and it is truly spectacular to watch.

We know that up to $25 \%$ of adults are needle phobic, which does unfortunately translates into increased morbidity and even increased mortality. For instance, needle-phobic teenagers are shown to be hesitant to seek out medical care. Those patients may postpone seeing a physician because they a worried about a blood draw or injection, until for instance a tumor might be too far spread. Several surveys found that a significant number of parents were hesitant to vaccinate their children because they could not stand the pain their child is going through. More than 350 children die of measles every day worldwide, most of them in resourcepoor countries, but in my career as a pediatrician I have seen five unvaccinated children die of measles in high-income countries. In other words, children are dying of preventable diseases because they or their parents are afraid of needle pain, yet the pain of a shot can now be prevented.

We now know that if we clinicians hold down a child for stitches, vaccinations, blood draws or other painful procedures, then many of those children need more analgesia, more anesthesia, and more man or woman power during their medical care for exactly the same procedure in future visits. So we in healthcare are creating children who are highly afraid of us and become needle phobic.

At Children's Minnesota, we are using lean quality improvement and our goal is to make sure these four 'non-negotiables' are offered more than $95 \%$ of the time. We have rolled this out over the last 3 years, unit by unit, department by department, meaning that every single time we include the front-line staff we ask them questions such as, "we need to get numbing cream on the child, what is the best way to do this?" We inquire about this, because whenever we want to change practice, the new way must be easier than the old way; otherwise, it will not be successful. This is why we have been successful in integrating all staff and implementing the four modalities [6].

Q Have you seen in the field that other pediatric pain centers are also taking this up? The evidence supporting the utilization of these four modalities is fairly clear. Much of this evidence has been around for 20 years or more, with a lot of this research coming from Canada. However, as with many things in medicine, there remains a chasm between research and what is put into practice. We recently received a grant from The Mayday Fund, a foundation which supports pediatric pain improvement, in order for us to roll out our initiatives in four other children's hospitals [16]. We have now trained a team from each of the four children's hospitals in Montréal, Atlanta, Toronto and Kansas City, and now we are in the process of visiting these hospitals and assisting the teams on the ground, and are using this funding to put this into practice as proof that we can replicate the roll-out of these modalities outside of Children's Minnesota.

A few hospitals worldwide have implemented versions of our (four non-negotiable) initiatives, but judging from the published literature usually it seems to be implemented only in some clinical areas and not system-wide for all children all of the time, including for vaccinations. Existing protocols often also unfortunately still omit the numbing cream. We feel strongly that right now we do not have enough research to undertake needle pokes without topical anesthesia. But there are several children's hospitals worldwide, especially the ChildKind certified hospitals, which have done hard work in the last years to really reduce pain system wide.

\section{Q The recently opened 'Healing} Environment' Pain, Palliative \& Integrative Medicine Clinic in Minneapolis has won several architecture awards \& represents a one-of-a-kind pain clinic; how does the healing space support advanced best clinical care for the large number of children with chronic pain?

As mentioned earlier, we have learned that taking pain away and providing excellent clinical care for children who are suffering from pain and other distressing symptoms is so such more than just prescribing the right medications. We have noticed that the space the children are in has a huge impact on how families are doing, the level of anxiety and the child's ability to heal.

A clinic space like ours, the "Kiran Stordalen and Horst Rechelbacher Pediatric Pain, Palliative and Integrated Medicine Clinic," to the best of our knowledge has never been built before [17]. It is absolutely unique, stunning and provides the means to offer light, sound and nature images so that children, even before 
they see the first clinician, can start to heal. In our clinic, at Children's Minnesota we see kids in pain, at the end of life and/or those who need integrative 'non-pharmacological' modalities. Having a well thought-out clinic architecture is yet another way to help children to heal beyond psychology, physical therapy, medications, and other modalities. Our clinic is an example of how we can utilize architecture and space to the best of its ability to create a healing environment. We heard feedback from our patients and their parents how uniquely helpful this is.

Q Do patients \& their parents play a larger role in designing treatment plans than in the past? If yes, what strategies have allowed them to become more involved?

An excellent piece of research explaining why our pain interventions may or may not be effective for an individual patient is from $\mathrm{Dr}$ Tracey's group in Oxford, UK, in 2011, where 22 volunteers were invited to carry out some pain testing [18]. This brilliant British group undertook a four step experiment: Step one, all of those 22 volunteers had an intravenous access receiving saline infusion, while pain testing was conducted. They asked the participants to put their hand on a research-approved hot plate and asked them how painful it was. Unsurprisingly, on average the reported pain was between 7 and 8 out of 10. Next, they told them beforehand that they wanted to find out whether giving the strong opioid remifentanil reduced the pain. They then gave the opioid without telling them and repeated the test, with the pain going down to around a 6 out of 10 . This showed that if you give patients a very strong opioid, it reduces the amount of pain. They then told them "I'm giving you the opioid now" and repeated the test; however, they had already given it. The pain went down to a 4 out of 10 . In other words, the positive expectation that an intervention is going to work more than doubled the treatment effect of this particular strong opioid. The fourth and last step of this experiment was to tell the volunteers that the research group had discontinued the opioid, and then repeated the hot-plate test and the pain went back to $7-8$ out of 10; however, they had not stopped the opioid. In other words, and this is what we see every single day, the patient's and parent's expectation of whether or not any of our pain intervention will work will predict to a large degree whether or not it is going to work.
Therefore, the best way to be successful in advanced pain treatment is to develop a plan together with our patients and their parents, which they believe will actually work. We have to be fairly convincing and offer choices. For example, if I expect my patient to do something, I may use some hypnotic language. I might say something like "would it be okay if I sit down and help you to be bothered by this pain less?" Or I might say something along the lines of "As we talked about it, I think you're ready to stop the intravenous morphine patient controlled analgesia. Do you think we should do this today or tomorrow?" Alternatively, I could suggest "I really expect you to learn one cool integrative modality, it could be hypnosis, biofeedback, deep breathing, etc." By going through these modalities very commonly you see the eyes of the child light up with a specific modality and they may ask, for instance, "what is hypnosis?" and then I might respond "well it is my favorite thing and you may love it too - would you like learning how to leave this room and go to your favorite place in the world in your imagination right now?" If the children or the parents have the opportunity choosing the integrative modality they believe works best, then it is much more likely to work. We often cannot be successful implementing a care plan for kids and parents without them buying into it.

\section{Q Do you have any closing comments or} messages for our readers?

In the USA we spend more than US\$560 billion annually on pain diagnosis and treatment, far more money than on cancer, kidney disease, and on cardiac diseases combined. Yet, some clinicians and especially most hospital administrations still believe incorrectly that pain in hospitalized children or children seeing their doctor is unavoidable and regards appropriate pain prevention as an afterthought. When we ask a child what is his or her number one worry when seeing a doctor would be, the child's answer would be pain caused by clinicians, especially by needles. If we ask parents, why they choosed to go to a hospital with their child and what were their top priorities? For parents, the number one priority is to find and treat the underlying condition. However, the second one on their list was to treat pain. For children, of course, it is the first priority - so pain prevention and treatment is far from an afterthought.

There is nothing worse for a parent to see their child being in pain. There is nothing worse for 
a child, than being in pain. We do know that pain has long-term negative consequences. We do know that even babies remember pain. Boys who have been circumcised without appropriate analgesia scream much longer and much harder at their 4-6 months vaccinations than boys who have been circumcised with appropriate analgesia. So of course infants remember this pain from a long time ago. We do know that unrelieved pain in hospitalized children increases their morbidity and even their mortality. We do know that children with unrelieved pain after trauma or burns have a much higher risk of developing post-traumatic stress disorder.

We, as the treating clinicians of children, have the responsibility to treat and prevent pain every single time. We do have knowledge and the resources, and we just need the will to actually do this. Analgesic treatment is mandatory for children when they undergo painful procedures and no avoidable suffering is acceptable nowadays, even for so-called minor interventions.

\section{Disclaimer}

The opinions expressed in this interview are those of the interviewee and do not necessarily reflect the views of Future Medicine Ltd.

Financial \& competing interests disclosure $S$ Friedrichsdorf is supported, in part, by the The Mayday Fund, NIH/National Cancer Institute, Children's Hospitals and Clinics of Minnesota Research Grant Program, NIH/National Institute of Nursing Research, and the Canadian Partnership Against Cancer. The author has no other relevant affiliations or financial involvement with any organization or entity with a financial interest in or financial conflict with the subject matter or materials discussed in the manuscript apart from those disclosed.

No writing assistance was utilized in the production of this manuscript.

\section{References}

1 International Association for the Study of Pain (IASP). Declaration of Montréal 2010. http://www.iasp-pain.org

2 Integrative medicine. www.childrensmn.org

3 Annual Paediatric Pain Master Class. http://noneedlesspain.org/ppmc/

4 Northwestern University Feinberg School of Medicine, Education in Palliative and End-of-Life Care. www.epec.net/

5 Child Kind International. www.childkindinternational.org

6 Friedrichsdorf SJ, Eull D, Weidner C. A Children's Comfort Promise: how can we do everything possible to prevent and treat pain in children using quality improvement strategies? Pediatr. Pain Lett.18(3), 26-30 (2016).

7 Children's Comfort Promise. www.childrensMN.org/comfortpromise
8 Friedrichsdorf SJ, Postier A, Eull D et al. Pain outcomes in a US children's hospital: a prospective cross-sectional survey. Hosp. Pediatr. 5(1), 18-26 (2015).

9 Taddio A, Appleton M, Bortolussi R et al. Reducing the pain of childhood vaccination: an evidence-based clinical practice guideline. CMAJ 182(18), E843-855 (2010).

10 Taddio A, Parikh C, Yoon EW et al. Impact of parent-directed education on parental use of pain treatments during routine infant vaccinations: a cluster randomized trial. Pain 156(1), 185-191 (2015).

11 Taddio A, Shah V, McMurtry CM et al. Procedural and physical interventions for vaccine injections: systematic review of randomized controlled trials and quasirandomized controlled trials. Clin. J. Pain 31(10 Suppl.), S20-S37 (2015).

12 Canadian Pediatric Society. Reduce the Pain of Vaccination in Babies. http://www.caringforkids.cps.ca
13 Canada Immunization. Reduce the Pain of Vaccination in Kids and Teens. http://www.immunize.ca

14 Karlson K, Darcy L, Enskär K. The use of restraint is never supportive (poster). Presented at: Nordic Society of Pediatric Hematology/ Oncology (NOPHO) 34th Annual meeting 2016 and 11th Biannual Meeting of Nordic Society of Pediatric Oncology Nurses (NOBOS). Reykjavik, Iceland, 27-31 May 2016.

15 Reducing pain with needle procedures. www.childrensmn.org

16 The May Day Fund. www.maydayfund.org/

17 Kiran Stordalen and Horst Rechelbacher Paediatric Pain, Palliative and Integrative Medicine Clinic Tour. https://vimeo.com/122654881

18 Bingel U, Wanigasekera $\mathrm{V}$, Wiech $\mathrm{K}$ et al. The effect of treatment expectation on drug efficacy: imaging the analgesic benefit of the opioid remifentanil. Sci. Transl. Med. 3(70), 70ra14 (2011). 